\title{
Selection against somatic parasitism can maintain allorecognition in fungi
}

\author{
7 Q1 Tamas Czaran ${ }^{\mathrm{a}}$, Rolf F. Hoekstra ${ }^{\mathrm{b}, *}$, Duur K. Aanen ${ }^{\mathrm{b}}$ \\ 8 Q2 a MTA-ELTE Research Group of Theoretical Biology and Evolutionary Ecology, Pázmány Péter sétány 1/C, 1117 Budapest, Hungary \\ 9 baboratory of Genetics, Wageningen University, Droevendaalsesteeg 1, 6708 PB Wageningen, The Netherlands
}

\section{A R T I C L E I N F O}

\section{Article history:}

Received 7 July 2014

Accepted 29 September 2014

Available online $\mathrm{xxxx}$

\section{Keywords:}

Cheating

Fungi

Heterokaryon incompatibility

Kin selection

Levels of selection

Somatic incompatibility

\begin{abstract}
A B S T R A C T
Fusion between multicellular individuals is possible in many organisms with modular, indeterminate growth, such as marine invertebrates and fungi. Although fusion may provide various benefits, fusion usually is restricted to close relatives by allorecognition, also called heterokaryon or somatic incompatibility in fungi. A possible selective explanation for allorecognition is protection against somatic parasites. Such mutants contribute less to colony functions but more to reproduction. However, previous models testing this idea have failed to explain the high diversity of allorecognition alleles in nature. These models did not, however, consider the possible role of spatial structure. We model the joint evolution of allorecognition and somatic parasitism in a multicellular organism resembling an asexual ascomycete fungus in a spatially explicit simulation. In a 1000-by-1000 grid, neighbouring individuals can fuse, but only if they have the same allotype. Fusion with a parasitic individual decreases the total reproductive output of the fused individuals, but the parasite compensates for this individual-level fitness reduction by a disproportional share of the offspring. Allorecognition prevents the invasion of somatic parasites, and vice versa, mutation towards somatic parasitism provides the selective conditions for extensive allorecognition diversity. On the one hand, if allorecognition diversity did not build up fast enough, somatic parasites went to fixation; conversely, once parasites had gone to fixation no allorecognition diversity built up. On the other hand, the mere threat of parasitism could select for high allorecognition diversity, preventing invasion of somatic parasites. Moderate population viscosity combined with weak global dispersal was optimal for the joint evolution of allorecognition and protection against parasitism. Our results are consistent with the widespread occurrence of allorecognition in fungi and the low degree of somatic parasitism. We discuss the implications of our results for allorecognition in other organism groups.
\end{abstract}

(C) 2014 Published by Elsevier Inc.
Q3 * Corresponding author.

E-mail addresses: czaran@caesar.elte.hu (T. Czaran), hoekstra.rf@gmail.com (R.F. Hoekstra), duur.aanen@wur.nl (D.K. Aanen) slime moulds (Hirose et al., 2011; Strassmann et al., 2011) and sessile marine invertebrates (Grosberg, 1988). However, the origin and maintenance of polymorphic genetic recognition cues remain incompletely understood despite substantial theoretical and empirical research (e.g. (Crozier, 1986; Nauta and Hoekstra, 1994; Rousset and Roze, 2007)). In this paper, we address the evolution of a specific example of kin recognition, allorecognition in multicellular (filamentous) fungi.

A multicellular individual essentially is a colony of cells, which cooperate to increase their inclusive fitness, for example by division of labour or by size-related protection against predation (Buss, 1987; Gavrilets, 2010; Ispolatov et al., 2012; Koschwanez et al., 2011). Extant multicellular organisms represent different stages in the transition towards individuality (Queller and Strassmann, 2009). In the most derived forms, the multicellular individual has become the new unit of selection, as adaptations at this level, such as an early germline-soma differentiation, render 
somatic cells evolutionarily dead ends (Bourke, 2011; Buss, 1987; Frank, 2003; Okasha, 2006). However, organisms with indeterminate growth, such as fungi, do not have an early germ-soma differentiation, so that all body parts retain the potential to reproduce and therefore still are "hopeful reproductives" (Aanen et al., 2008). Fungi further differ from other multicellular organisms in their growth mode. They form filaments (hyphae) that are branching and fusing regularly to form a dense, radially growing, network called the mycelium. Each fragment can reproduce via fission or the formation of asexual spores. In contrast to most other multicellular organisms, cell compartmentalization is not very strong and in some fungi nuclei can freely move through parts of the mycelium (Roper et al., 2011). Therefore, the cooperating units in the fungal colony are the haploid nuclei (Rayner, 1991). Colony size can be increased through hyphal fusion between germinating spores during colony establishment and between the hyphae of mature colonies (Read et al., 2010; Roca et al., 2005). Fusion between mycelia can be mutually beneficial (Aanen et al., 2009; Bastiaans et al., submitted for publication; Pontecorvo, 1958; Richard et al., 2012).

In spite of the potential benefits of fusion between individuals, fusion between different mycelia is restricted by genetic allorecognition systems based on gene polymorphisms at several loci, restricting fusion almost exclusively to clonally related colonies (Aanen, 2010; Glass et al., 2000; Saupe, 2000; Saupe et al., 2000). Successful fusion between colonies requires matching at all recognition loci otherwise mycelia are somatically incompatible and fusion will be interrupted by programmed cell death of the fused hyphal compartments at the border of two colonies. The widespread occurrence of allorecognition suggests that the disadvantages of fusion on average will be greater than the benefits (Nauta and Hoekstra, 1994). The most generally accepted hypothesis is that allorecognition has evolved to limit the opportunities for somatic parasites (e.g. (Aanen et al., 2008; Buss, 1982, 1987; Buss and Green, 1985; Grafen, 1990; Grosberg and Strathmann, 2007; Nauta and Hoekstra, 1994; Rousset and Roze, 2007). A somatic parasite is a variant that contributes less to colony functions, but relatively more to reproduction. Within a colony of cooperating nuclei, such a variant will be selected, but selection among colonies will disfavour such a variant. Thus, it is a cheater as it increases its relative fitness within a colony of wildtype nuclei, but does so at the cost of colony fitness (Ghoul et al., 2014). Although such mutants are not common in fungi, a few examples are known (Davis, 1960; Pittenger and Brawner, 1961).

Although it makes intuitive sense that allorecognition has evolved as a protection against somatic parasitism, its evolution is not well understood. First, Crozier (1986) pointed out that short-term selection will work against the genetic diversity of cues required for allorecognition. If fusion provides a benefit, or if rejection is costly, the common allele will always be favored, because it will fuse more often than rare alleles. Therefore, allorecognition 'eats up' the genetic variation upon which it crucially relies, a prediction now known as 'Crozier's paradox' (Aanen et al., 2008; Crozier, 1986; Rousset and Roze, 2007). The balance between short-term positive frequency-dependent selection limiting allorecognition diversity, as predicted by Crozier, and the long-term risk to be hit by a somatic parasite (or a 'cheat'; (Ghoul et al., 2014; Grafen, 1990), selecting for increased allorecognition diversity, thus remains unknown. Second, even under the assumption of potentially negative fitness consequences of somatic fusion, theoretical modelling predicts only limited polymorphism for allorecognition, and cannot explain the extreme extent to which polymorphism can go in many cases (Grosberg and Quinn, 1989; Jansen and van Baalen, 2006; Nauta and Hoekstra, 1994). Although the Nauta and Hoekstra model could explain the maintenance of a limited number of allotypes once they were already above a certain threshold frequency in the population, it could not explain the invasion of new allotypes starting from very low frequencies. However, this model did not take spatial structure into account.

In the present study, we test the hypothesis that the potential for somatic parasitism can select for allorecognition, and vice versa, that allorecognition keeps somatic parasites at a low frequency, using a spatially explicit model. We model the joint evolution of allorecognition and somatic parasitism in an asexual multicellular ascomycete fungus with the potential for somatic fusion. In our model, initially no parasitism and no allorecognition exist, i.e. every individual can fuse with every other. Mutation at the parasite locus can generate a nuclear parasite, while mutation at the allorecognition locus can generate new allorecognition types (allotypes). Somatic fusion is only possible between individuals with identical allotypes. The parasitic allele may spread horizontally by somatic fusion in compatible inter-individual confrontations. We systematically assess the effect of different assumptions about the details of parasitism and about the fitness consequences of fusion, and especially about the effect of spatial structure on both the allorecognition diversity and the level of parasitism evolving. Our study shows that allorecognition contributes to the stability of multicellular growth by preventing the invasion of somatic parasites, and vice versa, that the potential for somatic parasitism can select for extensive allorecognition diversity, thus solving Crozier's paradox.

\section{Methods}

\subsection{The model}

The model is a spatially explicit cellular automaton (CA) with which we addressed the joint evolution of allorecognition and somatic parasitism in a modular, sedentary multicellular organism, which produces propagules, such as spores (Fig. 1). However since application to other biological systems in which somatic fusion occurs is straightforward - we will use a more general terminology in the text throughout.

The basic assumptions of the model are the following:

1. Multicellular individuals are sedentary and occupy a 2D habitat represented by a $1000 \times 1000$ square lattice of toroidal topology. Each site of the lattice harbours one multicellular individual.

2. The organisms are haploid and reproduction is exclusively asexual through mitotic propagule formation. Therefore, we can simplify the genetic specification of the allorecognition system by assuming a single locus with a maximum of 50 different alleles. Thus the population contains 50 different allorecognition types or allotypes at most.

3. The individuals are identical in all but two respects: they may carry different alleles at the allorecognition locus, and either a parasitic $(h)$ or a non-parasitic $(H)$ allele at a "Parasitism" locus (which can be a functionally connected set of loci, of course). Every allorecognition allele can mutate with small probability $\left(10^{-6}\right.$ per generation) into any other one of the 49 . Also, a non-parasitic $H$ individual may produce a mutant parasitic $h$ offspring with probability $10^{-6}$ per generation; no back mutation from parasites to non-parasites is allowed. We have also tested a tenfold higher mutation rate towards parasitism $\left(10^{-5}\right.$ per generation).

4. Neighbouring individuals can fuse if they have the same allorecognition allele. Such fusions may result in extended chimaeric individuals that occupy more than one patch, but the actual effects of fusion remain local - each individual component in the chimaera feels the effect of fusion with 


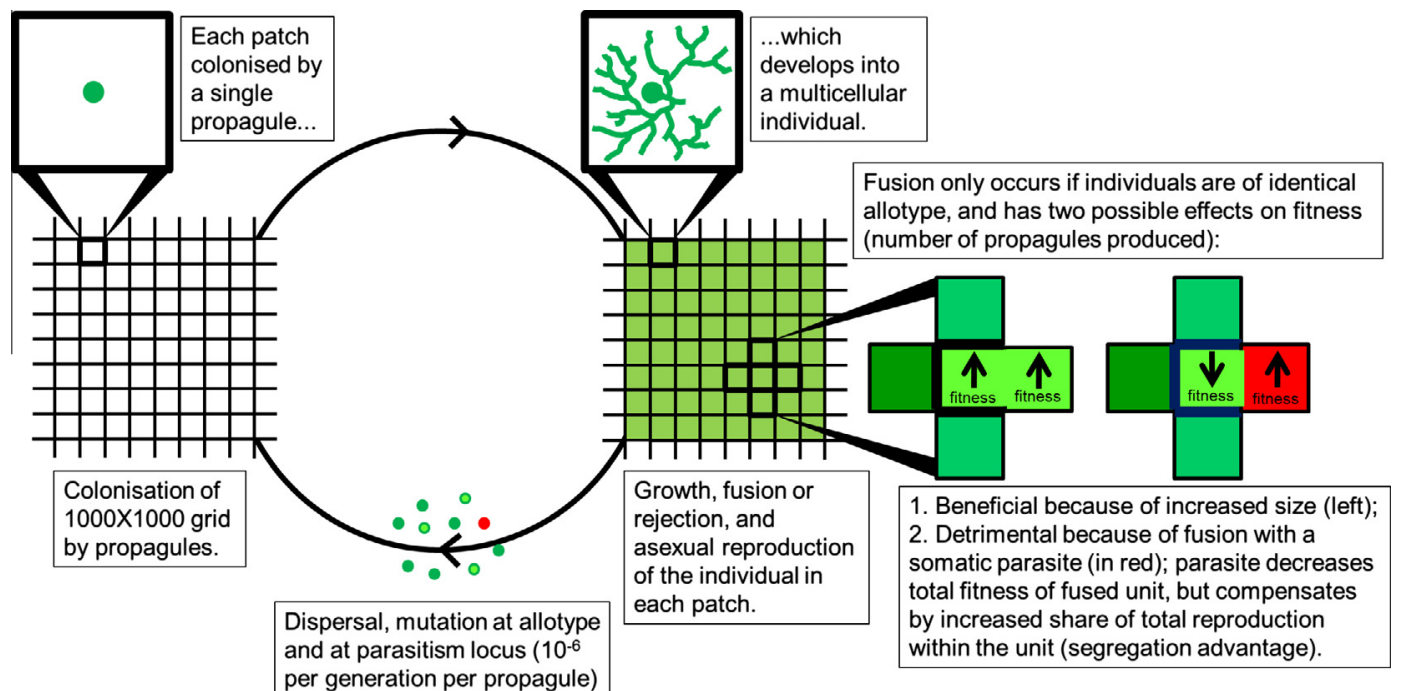

just its four immediate neighbours (i.e., within its Neumann neighbourhood). For extended individuals that occupy more than one patch, all calculated fitness values are scaled to the propagule output of a solitary non-parasitic individual in a single patch.

One possible effect of fusion is a fitness benefit (in terms of propagule production) depending on the number of fused neighbours according to a "diminishing returns" scheme, i.e. the more neighbours a certain individual is fused with the higher its fitness, but the fitness gain becomes smaller with each additional individual fused to the assembly:

$W^{\prime}=W_{0}+\beta \frac{N+n-1}{N+n}$,

where $W^{\prime}$ is the fitness of a focal individual or individual component (occupying one patch in the grid) after fusion, $W_{0}$ is the fitness of a solitary non-parasitic individual, $\beta$ is the fitness gain parameter, and $N$ and $n$ are the numbers of non-parasitic (" $H$ ") and parasitic (" $h$ ") neighbours, respectively, having the allotype of the focal individual. Note that $N+n \geqslant 1$, as the focal individual is always part of its own neighbourhood, and that the fitness benefit of fusion itself does not depend on the fused individuals being parasitic or non-parasitic.

5. The other effect of fusion is a possible fitness loss due to parasitism: parasitic "h" alleles decrease the (local) fitness of the individual they invade, and this cost of parasitism is a linear function of the proportion of parasitic individuals among those making up the chimaera. With this assumption the fitness $W$ (scaled per patch in the grid) of a fused unit containing $N$ non-parasitic and $n$ parasitic individuals is.

$W=W^{\prime}\left[\frac{N+(1-s) n}{N+n}\right]=\left[W_{0}+\beta \frac{N+n-1}{N+n}\right]\left[\frac{N+(1-s) n}{N+n}\right]$

$s$ is the fitness cost of parasitism; $0 \leqslant s \leqslant 1$. We shall use the term "Codominant" to label this linear algorithm of fitness phenotype determination, noting that this terminology is admittedly somewhat sloppy, since our model organism is not a diploid one. This algorithm is different from that of Nauta and Hoekstra (1994), who consider an all-or-none (step) type fitness cost function: fused units pay the cost of parasitism only if they consist entirely of parasitic individuals, i.e.,
$W=W^{\prime} \quad$ if $N \neq 0$

$W=W^{\prime}(1-s)$ if $N=0$.

This scenario will be referred to as "Recessive". For comparison we also use another step function for the cost of parasitism, assuming that even a single parasitic individual in the fused unit is sufficient for the entire fitness cost to apply:

$W=W^{\prime} \quad$ if $n=0$

$W=W^{\prime}(1-s)$ if $n \neq 0$.

This will be called the "Dominant" algorithm. We have repeated our simulations with all these three cost functions.

6. In a chimaeric individual containing fused parasitic and non-parasitic parts, parasitic $h$ alleles gain a disproportionally higher chance than non-parasitic $H$ alleles of being transmitted during reproduction. The measure of this segregation distortion is $\Theta$, the proportion of $h$ propagules in the yield of a chimaeric individual with equal numbers of $H$ and $h$ alleles $(\Theta>0.5)$, so that the relative abundances of non-parasitic $\left(W_{H}\right)$ and parasitic $\left(W_{h}\right)$ propagules produced by the focal individual (given in units of the propagule output of a solitary non-parasitic individual) are

$W_{H}=W \frac{(1-\Theta) N}{(1-\Theta) N+\Theta n}$

$W_{h}=W \frac{\Theta n}{(1-\Theta) N+\Theta n}$

7. The propagules are dispersed evenly over the sites within a certain distance from the mother-individual, i.e. propagule dispersal is local and scalable. We used local dispersal neighbourhoods of sizes $3 \times 3,11 \times 11$ and $21 \times 21$ sites ( $D=1,5$ and 10, respectively; $D$ is the radius of the dispersal neighbourhood). A small fraction $g$ of the propagules is dispersed "globally": all the sites of the lattice have equal chance to receive a globally dispersed propagule. The effect of global dispersal was tested at $g=0 \%, 1 \%, 5 \%$ and $10 \%$.

8. Propagule production is followed by the death of all mother individuals - at each site the next generation is started up from a propagule, which is randomly chosen from among those present at that site. 
Diversity was measured using the Gini-Simpson Index (Jost, 2006), which is the probability that two individuals taken from the population at random belong to different groups.

\section{Results}

Starting all simulations from a single allorecognition type with only non-parasitic genotypes, and allowing for rare (of probability $10^{-6}$ per individual per generation) mutations at the allorecognition locus and at the parasite locus, we ask two questions:

1. How many different allorecognition specificities do evolve depending on the various parameters (the fitness benefit of fusion $\beta$, the type and the parameter $s$ of the fitness cost function, the segregation distortion $\Theta$ and propagule dispersal distance $D)$ ?

2. Under what combinations of these parameters does the system expel the parasitic $h$ allele, maintain $H / h$ polymorphism, or drive the $H$ allele to extinction?

We have explored the biologically feasible part of the parameter space of the model, in search for possible patterns of polymorphism regarding both allorecognition and somatic parasitism. A represen- tative "walk" in the parameter space is given in Fig. 2. A more systematic scan is given in Figs. S1-S3 in the Supplement. One of the general observations is that the long-term coexistence of parasites and non-parasites is very improbable in this model. Any long-term polymorphism for parasitism we could find was temporal, i.e., oscillatory, and even that was confined to a very small part of the parameter space. Wherever we could see coexistence of parasites and non-parasites at generation 10,000 (like in the case of Fig. 2a2), longer simulations proved that coexistence was transitory - either parasites or non-parasites take over sooner or later. Another conspicuous feature of the results is that if the non-parasitic $H$ allele defeats the parasitic $h$ allele, it does so thanks to the selection of new allorecognition mutations, sometimes many of them, resulting in a high allotype diversity (Fig. 2). In some of the cases when the parasite takes over, the final state of the population is still polymorphic for allorecognition (like in Fig. 2b1). This might be considered as the remnant of past, failed attempts of non-parasitic strains to dispose of their parasitic mutants.

We will first consider the consequences of applying the three different cost-of-parasitism algorithms and of changing key parameters of the model before we explore the effect of spatial structure on the coevolution between allotype diversity and somatic parasitism.
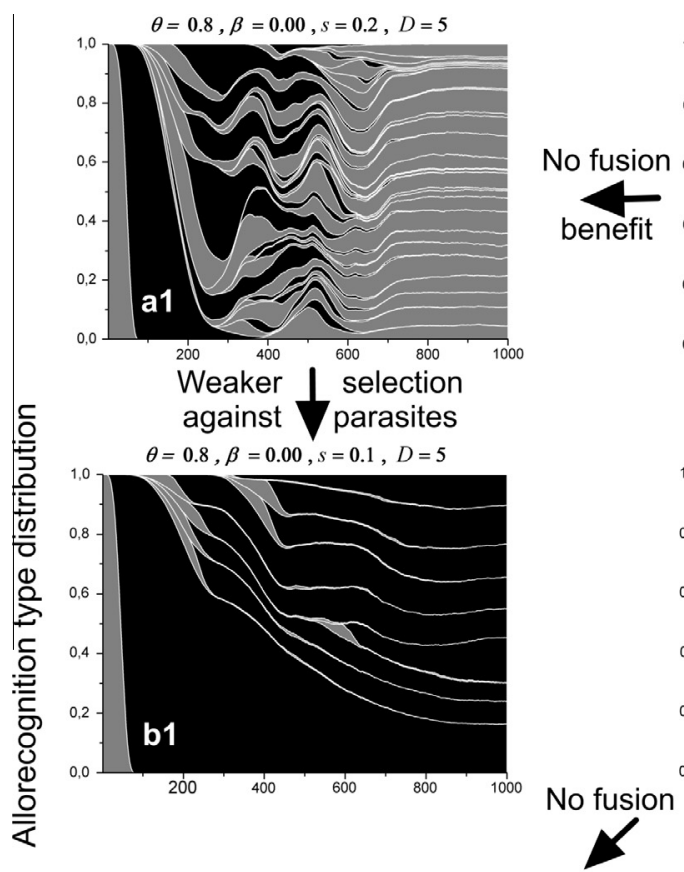

$\theta=0.8, \beta=0.00, s=0.2, D=10$

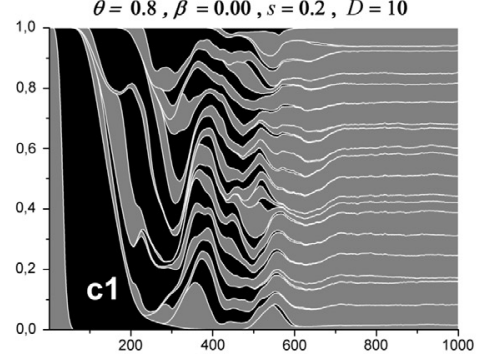

benefit

$\theta=0.8, \beta=0.10, s=0.2, D=5$
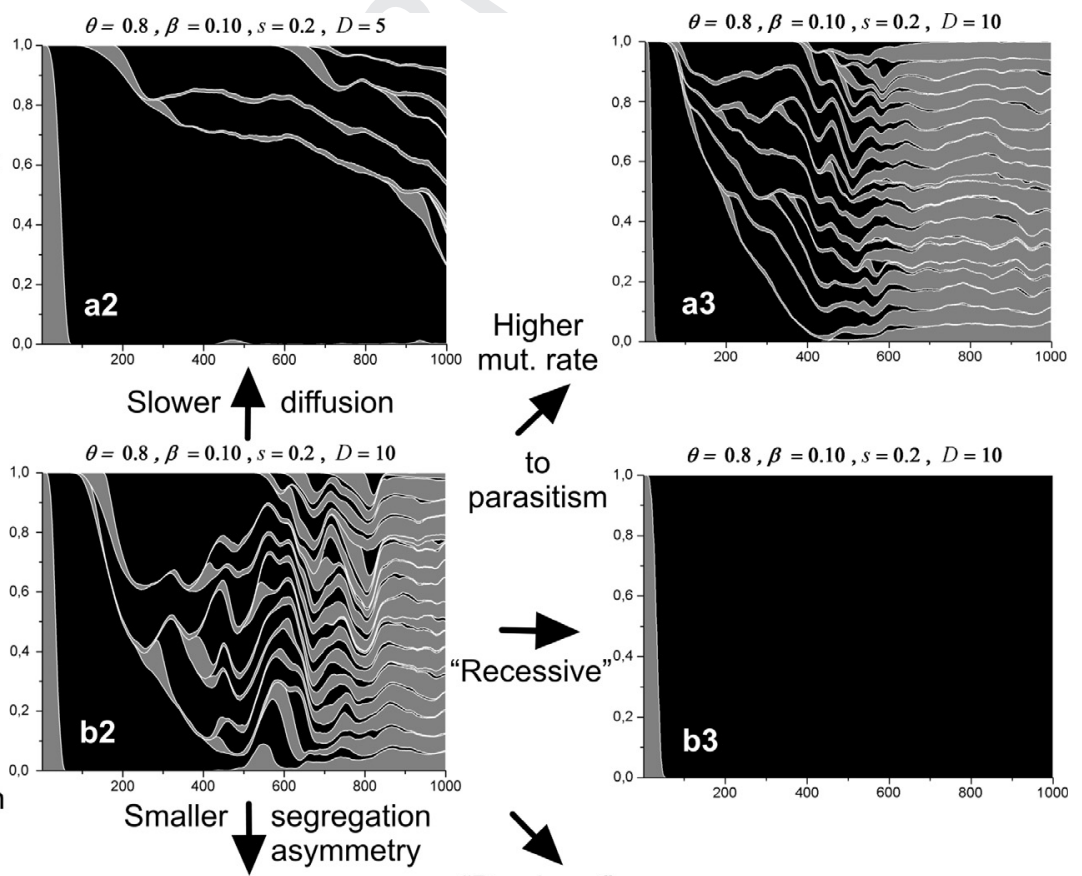

$\theta=0.6, \beta=0.10, s=0.2, D=10$
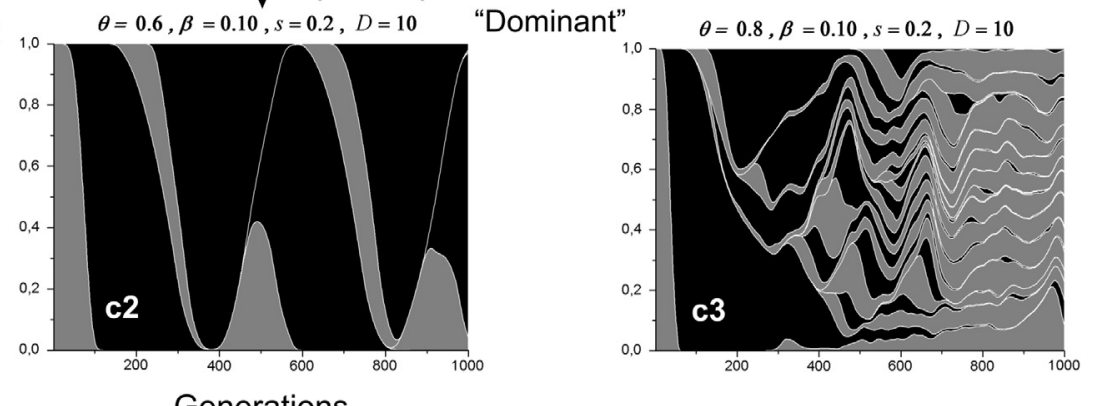

Generations

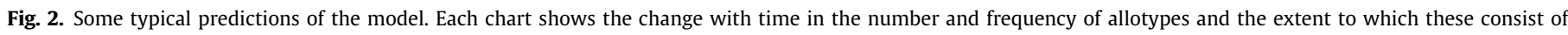

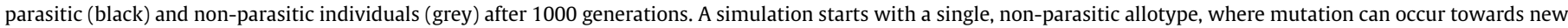

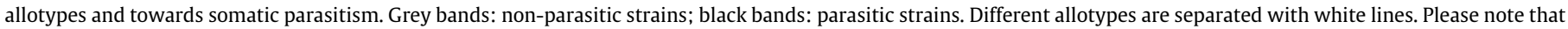

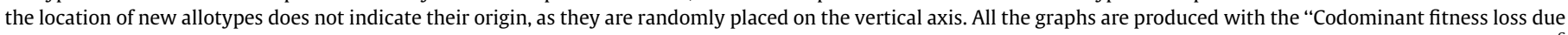

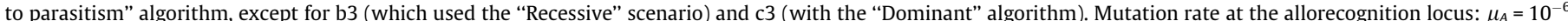

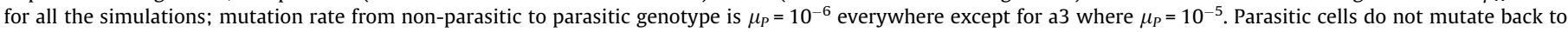
non-parasitic. All other simulation parameter values are specified on the graphs. For a systematic scan of the parameter space see the Supplement Figs. S1-S3. 


\subsection{The shape of the cost-of-parasitism function}

The shape of the cost-of-parasitism function has a clear effect on the coevolution of allorecognition and somatic parasitism: the "Recessive" step function (which represents the weakest selection against parasitism) yields high parasite frequencies and a low number of allotypes - in fact no new allotypes evolve and all cells become parasitic in most of the "Recessive" cases, at least if fusion itself carries a fitness benefit (Fig. 2b3; S1). Linear ("Codominant") fitness loss due to parasitism results in a high diversity of allotypes (Fig. 2b2; S2), but only at high segregation asymmetry $(\Theta=0.8)$ and mostly at stronger selection against parasitism $(s=0.2)$. At low segregation asymmetry $(\Theta=0.6)$ and relatively intensive spatial mixing $(D \geqslant 5)$ the system tends to oscillate, with only two allotypes present in the lattice at any point of time (Fig. 2c2), which seems to be a structurally unstable property of the model: even small changes in parameter values annihilate these oscillations. The diversity pattern of allorecognition types obtained with the "Dominant" scenario (step-like fitness-loss function dropping fitness to its minimum with a single parasitic individual in the fused unit) is qualitatively similar to that of the "Codominant" algorithm (Fig. 2c3; S3), except that no oscillations like on Fig. 2c2 show up. Relatively strong selection $(s=0.2)$ against parasitized units is a necessary condition for allotype diversity to build up, just like in the other two scenarios.

\subsection{Segregation distortion $(\Theta)$}

Segregation distortion has an interesting threefold effect on allorecognition evolution. Apart from the trivial tendency of parasites to be more likely to exclude non-parasites from the steady state of the simulations if $\Theta$ is larger, another, less obvious effect is that allorecognition diversity increases with $\Theta$ in all the cases where parasites are finally abolished (e.g., compare Fig. 2c2-b2, and panels A to B in Figs. S1-S3). At weaker counter-parasite selection $(s=0.1)$, increased segregation distortion always allows the parasite to take over, but the population might become persistently polymorphic on the allorecognition locus during the exclusion process of non-parasitic individuals (Fig. 2b1).

The surprising increase in the number of allorecognition types with increasing the segregation distortion $\Theta$ can be interpreted as an evolutionary reaction to the increased selection pressure from parasitic alleles: new non-parasitic allorecognition mutants enjoy the advantage of not immediately being parasitized, so they can spread as long as their population is small and therefore not likely to quickly produce its own parasitic mutant. That is, the populations of small and "clean" (parasite-free) allotypes persist and increase, and the larger the parasitic pressure (i.e., the larger $\Theta$ ) is, the higher their initial advantage will be relative to other, parasitized populations.

The third effect of increasing segregation distortion is that the frequency distribution of allotypes become more even. The more common an allotype becomes, the higher its chance to be hit by a parasitic mutant. This leads to frequency-dependent selection favouring rare allotypes, thus resulting in a frequency distribution of the mutant allotypes tending close to even (Nauta and Hoekstra, 1994). The larger $\Theta$ is, the more pronounced this frequency-equalizing effect seems to be, probably due to the consequent increased selection pressure from parasitism.

\subsection{The fitness benefit of fusion ( $\beta$ )}

We have compared the cases of zero $(\beta=0.0)$ and $10 \%(\beta=0.1)$ fitness advantage of fusion. The most conspicuous difference between these two parameter values was that, in the absence of a fusion benefit $(\beta=0.0)$, the chance of parasitic nuclei to take over was lower (Fig. 2a1and a3). That is, the advantage of parasitism increases with the fitness benefit of fusion (compare the left two columns to the right two columns of Figs. S1-S3). This may seem surprising at first, given that the fusion benefit is aspecific: it applies to the same extent to parasites and non-parasites. This result can be understood by considering that parasitic invasion can only occur through fusions; therefore, higher fitness rewards for fusion add an extra fitness bonus to parasites. This result can also be understood from the perspective of non-parasitic allotypes. As new, non-parasitic, allorecognition groups will initially be at a low frequency, they will have few partners to fuse with. If fusion is beneficial, this low starting frequency will hamper their invasion. As allotype diversity reduces the opportunities for parasites, lower allotype diversity increases the opportunities for parasites.

The advantage of parasitism due to the aspecific fitness reward of fusion can go so far that, in a considerable section of the parameter space, parasitic alleles become fixed at $\beta=0.1$, in contrast to the corresponding simulations with $\beta=0.0$ in which fusion is not beneficial in itself, and non-parasites win the game by evolving many allotypes (this can be clearly seen in Fig. S2B, for example).

\subsection{The local dispersal of propagules $(D)$}

The local dispersal of propagules (D) has a principal effect diagonally opposite to most previous model conclusions regarding parasitism (Frank, 1996). Conventional wisdom says that the parasite always does better if it can disperse efficiently within the host population(Hamilton and May, 1977). In contrast to this expectation, the great majority of our simulations show that it is the non-parasitic strain that performs better with increasing $D$. The general pattern seems to be that increasing dispersal increases the number of new allotypes invading the system, which then may or may not be followed by non-parasite takeover (compare Fig. 2a2-b2). A feasible explanation may be based on the fact that the fate of an allorecognition type depends on its ability to - at least temporarily escape from its own parasitic mutants. Parasitic mutants originate from non-parasites of the same allotype, and they can gain extra fitness by fusing with other individuals of their "mother strain", thus harvesting the fitness benefits due to fusion and also to segregation distortion. This can be achieved easily if dispersal does not drift the parasitic mutant far away from its mother strain. In fact dispersing far away is most probably fatal for new parasites, because with a very high probability they lose both sources of fitness gain (those from $\beta$ and from $\Theta$ ). Viewed from the non-parasites' aspect, dispersal is the best escape for a relatively rare allotype from its own parasitic mutants; it can keep the allotype free of parasites for a long time. If the cost of parasitism is low $(s=0.1)$ and segregation distortion is strong $(\Theta=0.8)$, the ultimate winner can still be the parasite. Also under these conditions, the more advantage was given to non-parasitic allotype mutants by dispersal at the beginning, the more parasitic allorecognition groups will be present in the stationary phase of the process (compare within-column panels of Figs. S1-S3). That is, allotype diversity is correlated to dispersal, even if parasites win the game finally.

The spatial patterns generated by fusion dynamics and limited propagule dispersal are almost always patchy to some extent. This is a direct consequence of two different effects: the aspecific benefit of fusion (wherever it is assumed) which selects for allotypes staying aggregated, and the crucial dependence of parasitic mutants on their ability to aggregate with their own hosts. The statistical effects of these forces on the distribution of local fusions are summarized in Fig. 3.

\subsection{The global dispersal of propagules ( $g$ )}

The global dispersal of propagules $(g)$ has proven to be of a twofold effect on allotype diversity: it may help or hinder the 

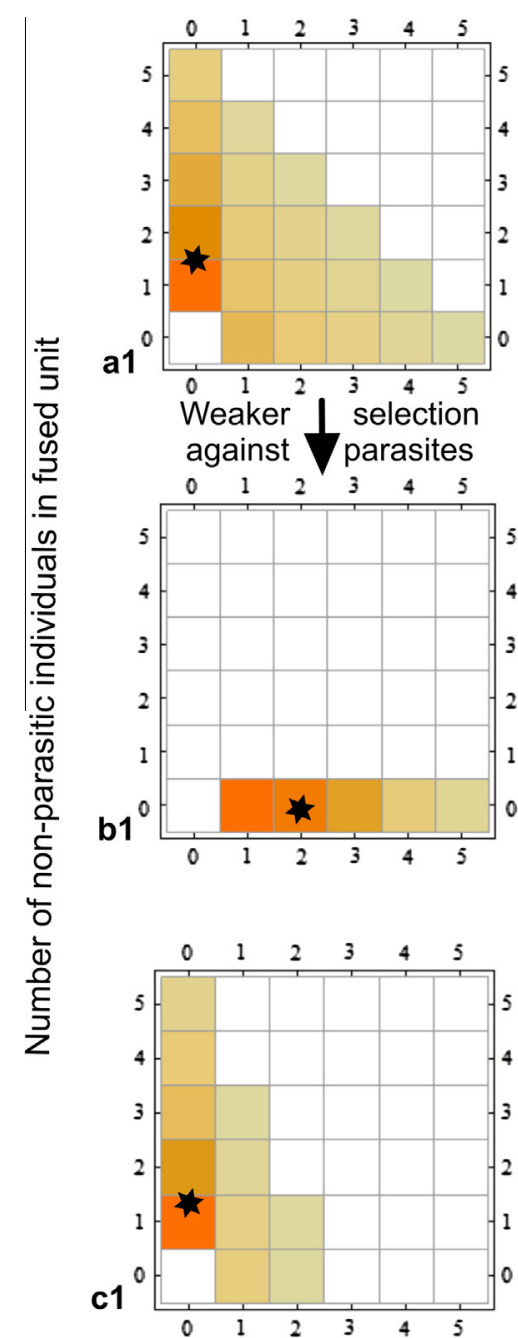
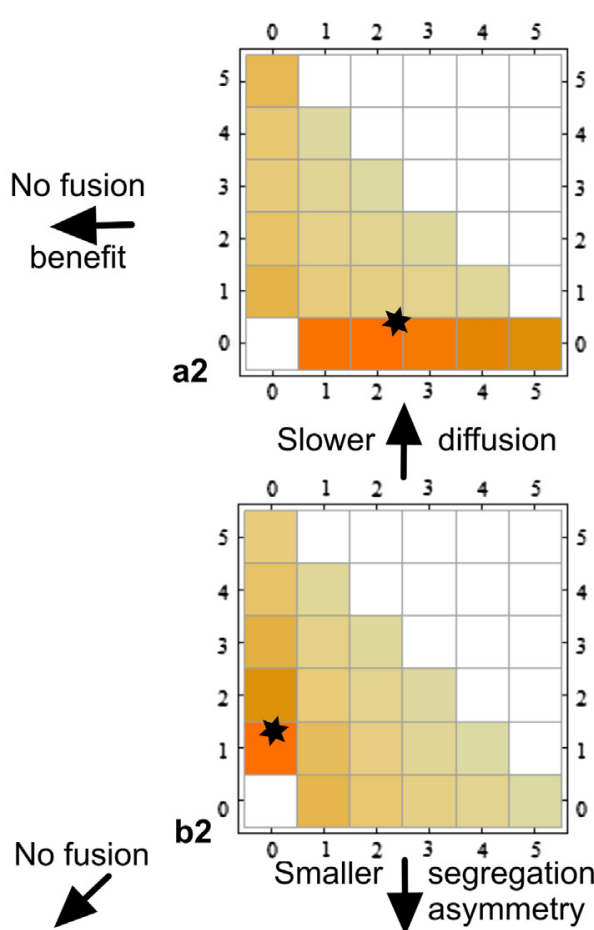

benefit

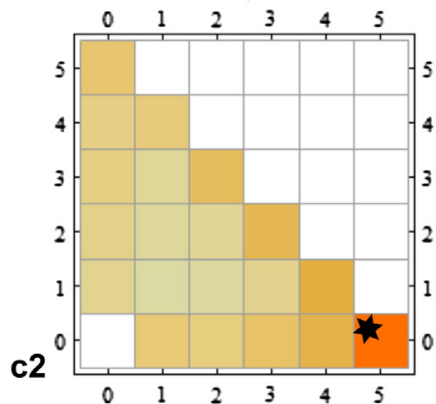

Higher mut. rate
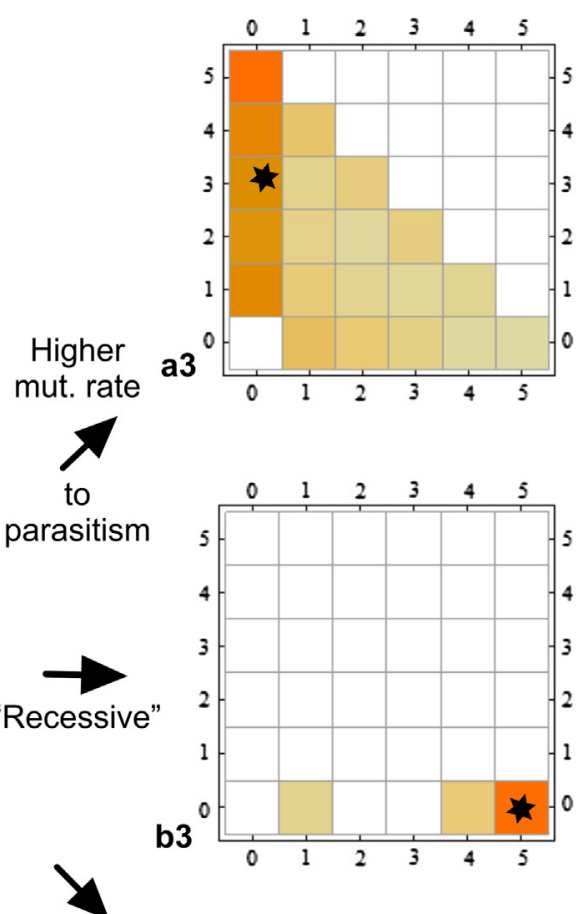

"Dominant"

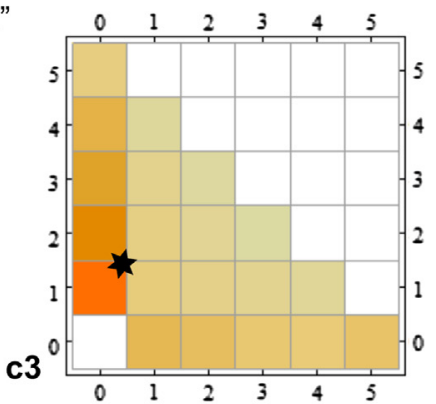

Number of parasitic individuals in fused unit

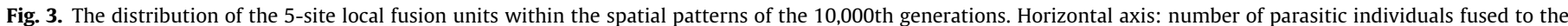

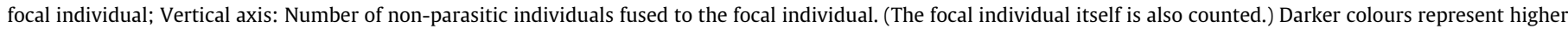

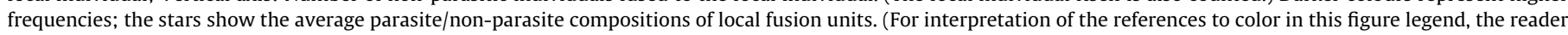
is referred to the web version of this article.)

evolution of new allotypes. Fig. 4 shows the effect of increasing global spore dispersal in the "Codominant" (probably the most realistic) series of simulations, for both segregation asymmetries studied $(\Theta=0.6$ and 0.8 ). It is obvious that for very high segregation asymmetry $(\Theta=0.8)$ global dispersal is always deleterious it helps parasites take over (Fig. 4a). For moderate asymmetry $(\Theta=0.6)$, however, weak global dispersal (at $g=1-5 \%$ ) is beneficial, but strong global dispersion $(g>5-10 \%)$ is also deleterious (Fig. 4b).

\section{Discussion}

Somatic fusion provides opportunities for somatic parasites. The overall conclusion of our study is that allorecognition can be an efficient means to prevent the spread of somatic parasites and vice versa, that the potential of parasitism can select for extensive allorecognition diversity. Thus, selection against somatic parasitism provides a solution to Crozier's paradox and explains the extensive allorecognition found in populations of fungal species and other organisms with modular growth.
4.1. A comparison with previous models on the coevolution of cooperation and allorecognition

In contrast to most other studies, our model assumes cooperation, viz. among the cells of a multicellular individual, as a starting point, and then considers the effect of cue-dependent fusion among individuals on the opportunities for somatic parasites. Other theoretical studies have sought to explain the evolution of cue-dependent cooperation bottom-up, i.e. starting with solitary behaviour (e.g. (Axelrod et al., 2004; Czaran and Hoekstra, 2009; Jansen and van Baalen, 2006; Lee et al., 2012; Rousset and Roze, 2007). Multicellular growth followed by possible fusion among multicellular individuals contains two layers of cooperation, i.e. altruistic cooperation among cells within the individual and mutually beneficial cooperation between fusing individuals. In our model, 'defecting' means 'parasitizing' because we assume a tradeoff between somatic parasitism and somatic growth (which we show is reasonable). Therefore, in contrast to previous models (Axelrod et al., 2004; Czaran and Hoekstra, 2009; Jansen and van Baalen, 2006; Lee et al., 2012; Rousset and Roze, 2007), a parasite has reduced basic fitness when it grows solitarily. 


\section{The effect of global spore dispersal on allotype evolution}

(a)

$$
\Theta=0.8, \beta=0.1, s=0.2
$$

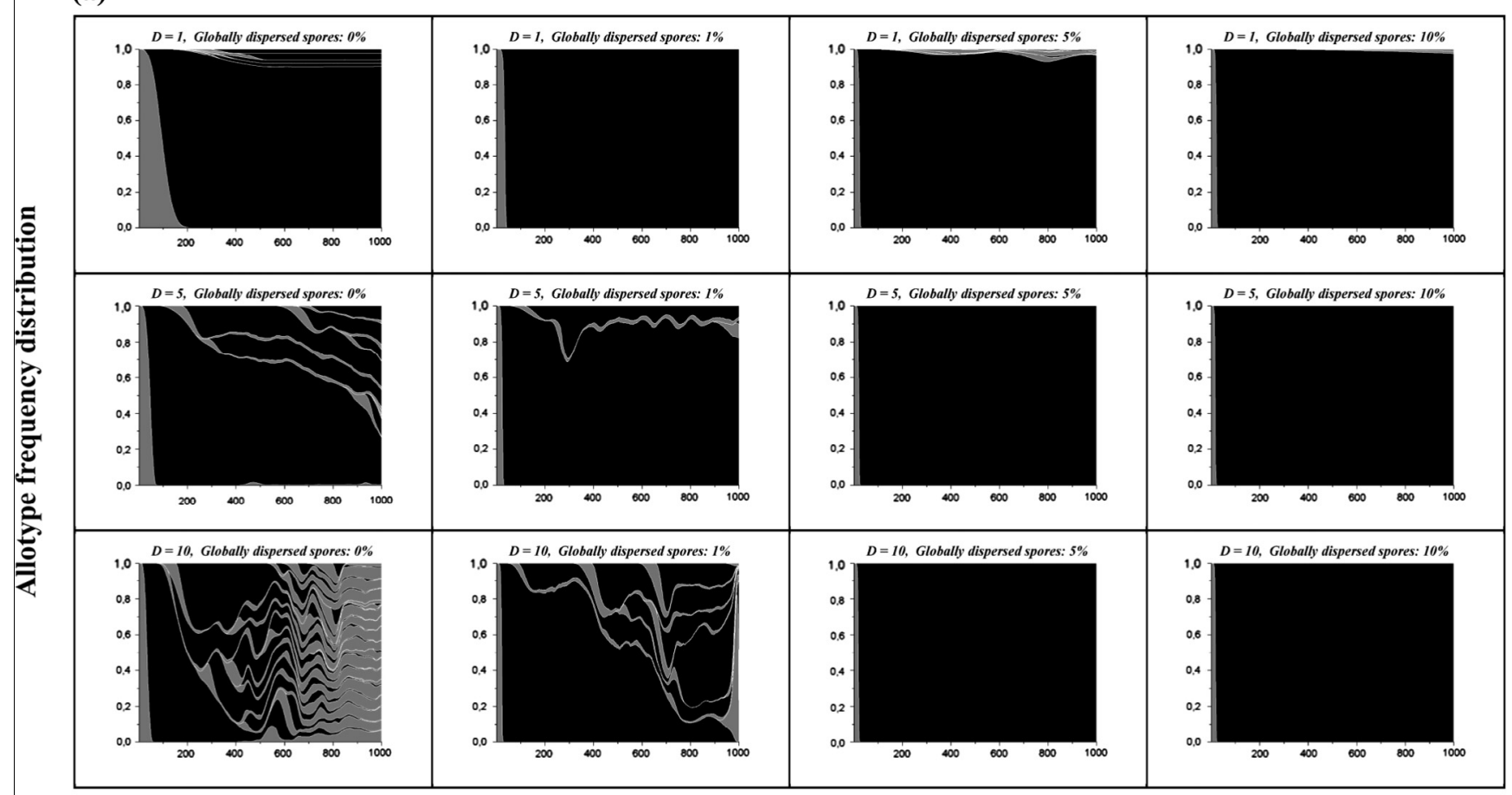

(b)

$\Theta=0.6, \beta=0.1, s=0.2$

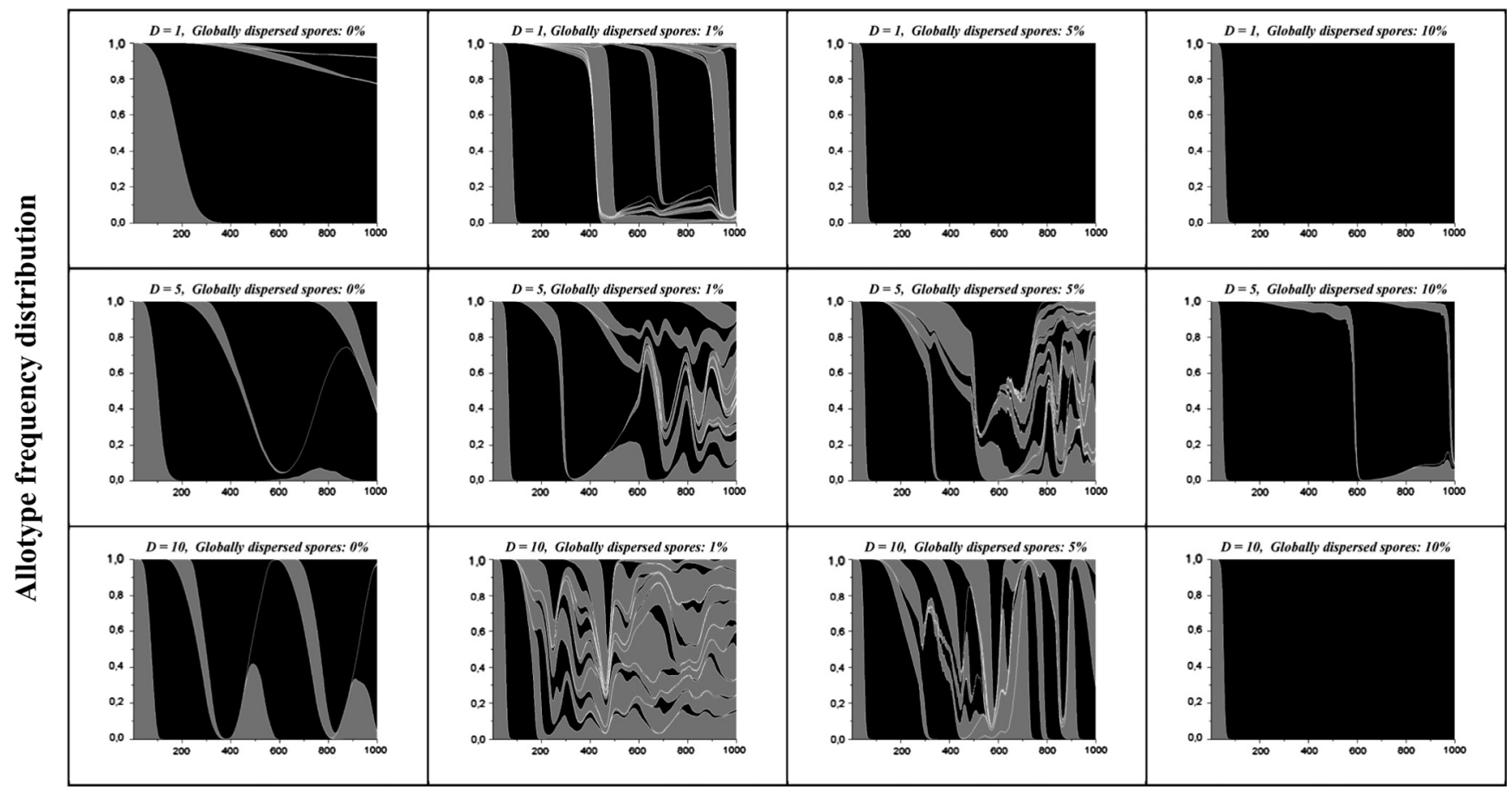

\section{Time (Generations)}

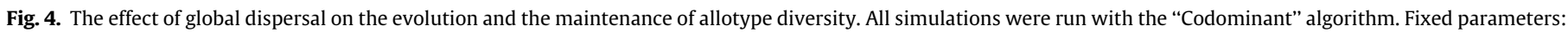

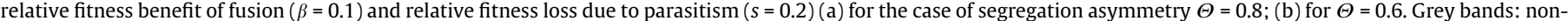
parasitic strains; black bands: parasitic strains.

Because of the theoretical problems to explain the maintenance of allorecognition diversity, it has been argued that allotype diversity could also be maintained if recognition loci have a secondary function that is subject to diversifying selection
(Crozier, 1986; Rousset and Roze, 2007). For example, if allorecognition loci also function in recognition of parasites (of a different species), they may be under diversifying selection because of coevolution with these (Paoletti and Saupe, 2009). Another 
possibility is disassortative mating based on allotypic differences, and a recent paper shows that this can stabilise allotype diversity (Holman et al., 2013). Although these hypotheses are not incompatible with the results of this paper, our results demonstrate that it is not necessary to infer secondary functions of allorecognition labels, although further research is needed to study the assumptions and parameter values of our model.

\subsection{The benefit of fusion}

We confirm Crozier's (Crozier, 1986) prediction that, if fusion is beneficial, short-term selection works against allorecognition diversity. This in turn increases the probability that the parasite takes over. Thus, selection in favour of fusion means selection against the invasion of new allorecognition types at the same time, because new allotypes prevent fusions and thus also the harvest of the benefit thereof. Since the chances of non-parasitic genotypes to exclude parasitic invasion depend on their ability to hitch-hike on new allorecognition mutants, any selective force that prevents new allotypes from spreading is advantageous for the parasite.

But is fusion between different individuals beneficial? The fact that in many groups of organisms somatic fusion, either between individuals or between tissues, is a physiological possibility does suggest that it is. Possible advantages of inter-individual fusion are genetic complementation, mitotic recombination or more effective resource utilization. Also fusion between genetically identical individuals has been shown to be advantageous, presumably due to more efficient reproduction with increased size (Aanen et al., 2009; Amar et al., 2008; Bastiaans et al., submitted for publication; Raymundo and Maypa, 2004; Richard et al., 2012). The benefit of fusion between individuals probably depends on size (Aanen et al., 2008; Amar et al., 2008). Experiments show that fusion is beneficial if individuals are small, but not if they are large (Bastiaans et al., submitted for publication). In our model, no benefit of fusion $(\beta=0)$ implies that a multicellular individual within one patch is large enough to yield the full benefit of multicellularity, so that additional increase in size because of between-individual fusion is neutral.

Alternatively, fusion between cells or hyphae within a single (physical) individual provides an advantage (Nauta and Hoekstra, 1994). Fusion among fragmented individuals of the same clone would then be an artefact of this intra-organismal fusion and hence a consequence of an imperfect non-self-recognition, rather than a kin-recognition system (Aanen et al., 2008). For fungi, the significance of intra-individual fusion has indeed been shown (Rayner, 1991; Rayner et al., 1984; Xiang et al., 2002). Also for colonial marine invertebrates, intra-individual fusion is probably important (Feldgarden and Yund, 1992; Hughes et al., 2004).

\subsection{The role of dispersal}

Previous models have shown that the evolution of allorecognition is difficult to understand in the absence of population structure(Grosberg and Quinn, 1989; Hartl et al., 1975; Nauta and Hoekstra, 1994; Rousset and Roze, 2007; Tsutsui, 2004). Here we analysed the effect of population viscosity, using three levels of local dispersion, and, for a subset, local dispersal in combination with varying levels of global dispersion. The relationship between dispersion and the evolution of allorecognition and parasitism was complex. Dispersal changes the balance between selection at two hierarchical levels, i.e. among nuclei within individuals, and among individuals. In general, some dispersal favours among-individual selection relative to within-individual selection, and thus favours allorecognition diversity, and disfavours parasitism. Furthermore, if fusion provides a benefit, dispersal affects the efficiency of positive frequency-dependent selection of allotypes.
In the simulations without any global dispersion, generally the most efficient local dispersion $(D=10)$ was optimal for the buildup of allorecognition diversity and selection against somatic parasitism (compare Figs. 2b2-2a2). This can be understood as follows. A new, mutant allotype free of parasites, freely spreads in the habitat until it produces its own parasite by mutation. With low dispersal, this parasitic mutant will remain close to its non-parasitic host allotype, so it has a good chance to exploit it. With more dispersal, this chance decreases. In addition, with long-distance dispersal the host individuals are more dispersed (they do not form contiguous patches), so that even if the parasite gets in contact with a few hosts, it will not have access to the whole host population. Once it has excluded all the hosts locally available, the parasite has a disadvantage compared to non-parasitic, individuals of other allotypes, with which it cannot fuse, and this is why it goes extinct.

The effect of some global dispersal upon the evolution of allorecognition diversity and somatic parasitism is subtle. On the one hand, global dispersal allows parasites to spread efficiently through the population, limiting the scope for parasite-free individuals to evolve a new allotype. A high percentage of global dispersion $(g \geqslant 10 \%)$ indeed always led to fixation of parasitism in the absence of any allotype diversity (Fig. 4). On the other hand, some global dispersal helps new non-parasitic allotype mutants find and invade parasitic patches of other allotypes, i.e., sites in which they have a fitness advantage compared to their parasitic neighbours.

Not much is known on the dispersal rates of relevant organisms in nature. Grosberg and Quinn (1986) found that in Botryllus schlosseri allorecognition types tend to cluster, more than if larval dispersal was random. This result has been confirmed in later studies, although rare dispersal further away also occurs (BenShlomo et al., 2008; Grosberg, 1987). However, actual dispersal may have been underestimated in these studies, because clean substrates were available close to a source colony. If nearby substrates are covered, larvae are forced to swim further, so that the actual dispersal rate will be higher than measured in these experiments (Grosberg, 1987). Social bacteria have been found to have strong population structure, even at small spatial scales, demonstrating that dispersal is limited (Vos and Velicer, 2008). In fungi, spores are generally wind-dispersed, which is highly efficient. Nevertheless, it has been shown that the great majority of spores will not disperse far $(<100 \mathrm{~m})$ from their origin (Lacey, 1996; Wolfenbarger, 1946). A recent study on spore dispersal in six ectomycorrhiza-forming basidiomycetes even showed that $95 \%$ of basidiospores fall within $58 \mathrm{~cm}$ of the cap (Galante et al., 2011).

\subsection{The nature of parasitism}

A few studies have addressed somatic parasitism in multicellular organisms. In the ascomycete fungus Neurospora crassa, which resembles the organism modelled in this paper, Pittenger and Brawner (1961) described a mutant that increased in frequency within the mycelium without an obvious effect on mycelium fitness, so that it is not a parasite as defined here. However, using an auxotrophic marker, this mutant artificially became a parasite. Under selective conditions, the mutant could only grow as a chimaera in combination with the wild-type nuclei due to the auxotrophy, but within the chimaera, the parasite increased in frequency up to the point where mycelial growth ceased. Davis (Davis, 1960) described a different parasite. From a pantothenate-requiring mutant $\left(\mathrm{Pan}^{-}\right)$, he isolated a mutant that could grow on low concentrations of pantothenate $\left(\mathrm{Pan}^{+}\right)$. A chimaera of these two genotypes could also grow on this low concentration, but during growth, the $\mathrm{Pan}^{-}$nuclei took over within the mycelium, reducing mycelial fitness up to the point where growth stopped. In these 
two studies, the effect of the parasites thus was most consistent with a linear cost-of-parasitism function, although in a slightly more complex fashion than modelled here.

De Boer (1995)) modelled the evolution of allorecognition as a means to protect 'the genetic integrity' of an individual. He argued that restriction of somatic fusion may function to preserve specific evolved adaptations. A particular individual adapted to one environment, may be maladapted to a different environment, and another individual vice versa. If these individuals fuse, the maladapted individual will be a parasite relative to the welladapted genotype, and these roles will be reverted in the other environment. Somatic parasitism, therefore, is a relative and not an absolute characteristic of an individual, depending on the environment and the individual with which it fuses. The examples on fungi above show that this indeed may be the case, as the effect of an auxotrophic mutation will depend on the fusing partner, and on the environment, i.e. on the presence of the nutrient. Crucially, these examples also show that for some mutants the costs saved on somatic functions, i.e. the uptake or production of nutrients, can be invested in personal reproductive success, making such mutants genuine parasites as modelled in this paper.

In Botryllus, heritable differences have been found between genotypes in fused individuals for replacement of germline and somatic cells (De Boer, 1995; Laird et al., 2005; Stoner et al., 1999; Stoner and Weissman, 1996). Note, however, that the modelled organism resembles a modular organism without a germsoma differentiation. Therefore, we do not distinguish between somatic and germline parasites, as has been done for the colonial protochordate B. schlosseri (Stoner et al., 1999).

Recently, Kuzdzal-Fick et al. (2011) experimentally selected for somatic parasitism under conditions of low relatedness among cells in Dictyostelium, where multicellular fruiting bodies form by aggregation of cells. In nature, relatedness among cells in this species is high via a combination of kin recognition and efficient dispersal of spores (Kuzdzal-Fick et al., 2011; Ostrowski et al., 2008). However, experimentally manipulated conditions of low relatedness favoured the evolution of obligate parasites, which could not produce fruiting bodies alone, but only in combination with non-parasites.

Not only nuclear parasitic genes can lower fitness of the multicellular individual, but also cytoplasmic parasites. For example, in fungi, suppressive mitochondria (Bertrand, 2000) and deleterious plasmids and viruses are widespread (Ghabrial and Suzuki, 2009). Such selfish genetic elements can infect other individuals following somatic fusion. Therefore, allorecognition not only can protect against infection with nuclear parasites (Debets and Griffiths, 1998), but potentially also against infection with cytoplasmic parasites (Brusini et al., 2011). Several studies have indeed found that allorecognition provides some protection against infection with cytoplasmic elements, although not perfect (Debets et al., 1994). Our model could also be regarded as covering the case of a parasitic cytoplasmic element, by assuming that $h$ individuals represent cytoplasmically infected individuals. Rare mutations to novel parasitic nuclei could then be interpreted as rare infections between individuals of different allorecognition type. To simulate that a fraction of the offspring of infected individuals can be cured, especially after sexual reproduction, we would need to extend our model to allow for mutations to an $H$ genotype in a propagule from an $h$ individual.

\subsection{Conclusion}

Our study shows that allorecognition can stabilise multicellularity in organisms with somatic fusion and vice versa, that the threat of somatic parasitism provides the selective conditions for the maintenance of allorecognition, thus solving Crozier's paradox.
Under many reasonable combinations of parameter values, we obtained a high degree of allorecognition, in combination with a very low degree, or absence, of parasitism. If fusion provides a benefit, the evolution of allorecognition requires more stringent conditions, confirming Crozier's (Crozier, 1986) prediction. Population viscosity with some long-range dispersal generally was most favourable for the evolution of allorecognition diversity and selection against parasitism.

Idiosyncrasies of specific organisms will determine the potential for somatic parasitism and the need for allorecognition. Future studies therefore need to address basic details of multicellular growth, somatic fusion and the scale of competition in different species under natural conditions. Furthermore, experimental studies are needed to measure the mutation rate towards parasitism and the characteristics of somatic parasites. For example, several loss-of-function mutations in fungi have been found to be somatic parasites in combination with wild-type genotypes (Davis, 1960). If somatic parasitism is a general characteristic of loss-of-function mutants, this increases the potential number of mutations causing somatic parasitism dramatically. Also the effect of parasitic mutants upon individual fitness, and whether this is 'dominant' or 'recessive' will need to be determined. This knowledge is necessary to validate our theoretical predictions for specific cases.

\section{Uncited reference}

Wilson and Grosberg (2004).

\section{Acknowledgments}

The authors thank Eric Bastiaans and Fons Debets for useful discussion, and Alan Grafen and Niels Anten for comments on the manuscript. DKA was funded by a grant of NWO (vidi) and TC by visitor's grants provided by NWO, PE\&RC and OTKA Grant No. K100806.

\section{Appendix A. Supplementary material}

Supplementary data associated with this article can be found, in the online version, at http://dx.doi.org/10.1016/j.fgb.2014.09.010.

\section{References}

Aanen, D.K. et al., 2008. The social evolution of somatic fusion. Bioessays 30, 11931203.

Aanen, D.K. et al., 2009. High symbiont relatedness stabilizes mutualistic cooperation in fungus-growing termites. Science 326, 1103-1106.

Aanen, D.K., Debets, A.J.M., Glass, N.L., Saupe, S.J., 2010. Biology and genetics of vegetative incompatibility in fungi. In: Borkovich, K., Ebbole, D.J., (Eds.), Cellular and Molecular Biology of Filamentous Fungi.

Amar, K.-O et al., 2008. Coral kin aggregations exhibit mixed allogenic reactions and enhanced fitness during early ontogeny. BMC Evol. Biol. 8.

Axelrod, R. et al., 2004. Altruism via kin-selection strategies that rely on arbitrary tags with which they coevolve. Evolution 58, 1833-1838.

Bastiaans, E., et al., submitted for publication. Experimental demonstration of the benefits of somatic fusion and the consequences for allorecognition. Evolution. Ben-Shlomo, R. et al., 2008. Pattern of settlement and natural chimerism in the colonial urochordate Botryllus schlosseri. Genetica 132, 51-58.

Bertrand, H., 2000. Role of mitochondrial DNA in the senescence and hypovirulence of fungi and potential for plant disease control. Ann. Rev. Phytopathol. 38, 397422.

Bijma, P., Wade, M.J., 2008. The joint effects of kin, multilevel selection and indirect genetic effects on response to genetic selection. J. Evol. Biol. 21, 1175-1188. Bourke, A.F.G., 2011. Principles of Social Evolution. Oxford University Press, Oxford.

Brusini, J. et al., 2011. Parasitism and maintenance of diversity in a fungal vegetative incompatibility system: the role of selection by deleterious cytoplasmic elements. Ecol. Lett. 14, 444-452.

Buss, L.W., 1982. Somatic cell parasitism and the evolution of somatic tissue compatibility. Proc. Natl. Acad. Sci. USA 79, 5337-5341.

Buss, L.W., 1987. The Evolution of Individuality. Princeton University Press, Princeton. 
Buss, L.W., Green, D.R., 1985. Histoincompatibility in vertebrates: the relict hypothesis. Dev. Comp. Immunol. 9, 191-201.

Charpentier, M.J.E. et al., 2007. Kin discrimination in juvenile mandrills, Mandrillus sphinx. Anim. Behav. 73, 37-45.

Chen, B.J.W. et al., 2012. Detect thy neighbor: identity recognition at the root level in plants. Plant Sci. 195, 157-167.

Crozier, R.H., 1986. Genetic clonal recognition abilities in marine invertebrates must be maintained by selection for something else. Evolution 40, 1100-1101.

Czaran, T., Hoekstra, R.F., 2009. Microbial communication, cooperation and cheating: quorum sensing drives the evolution of cooperation in bacteria. PLOS ONE 4.

Davis, R.H., 1960. Adaptation in pantothenate-requiring Neurospora. 2. Nuclear competition during adaptation. Am. J. Bot. 47, 648-654.

De Boer, R.J., 1995. The evolution of polymorphic compatibility molecules. Mol. Biol. Evol. 12, 494-502.

Debets, A.J.M., Griffiths, A.J.F., 1998. Polymorphism of het-genes prevents resource plundering in Neurospora crassa. Mycol. Res. 102, 1343-1349.

Debets, F. et al., 1994. Vegetative incompatibility in Neurospora - its effect on horizontal transfer of mitochondrial plasmids and senescence in natural populations. Curr. Gen. 26, 113-119.

Dudley, S.A., File, A.L., 2007. Kin recognition in an annual plant. Biol. Lett. 3, 435438.

Feldgarden, M., Yund, P.O., 1992. Allorecognition in colonial marine invertebrates does selection favor fusion with kin or fusion with self? Biol. Bull. 182, 155-158. Frank, S.A., 1996. Models of parasite virulence. Quart. Rev. Biol. 71, 37-78.

Frank, S.A., 2003. Perspective: repression of competition and the evolution of cooperation. Evolution 57, 693-705.

Galante, T.E. et al., 2011. 95\% of basidiospores fall within $1 \mathrm{~m}$ of the cap: a field- and modeling-based study. Mycologia 103, 1175-1183.

Gavrilets, S., 2010. Rapid transition towards the division of labor via evolution of developmental plasticity. PLOS Comput. Biol. 6.

Ghabrial, S.A., Suzuki, N., 2009. Viruses of plant pathogenic fungi. Annu. Rev. Phytopathol., 353-384.

Ghoul, M. et al., 2014. Toward an evolutionary definition of cheating. Evol. Int. J. Org. Evol. 68, 318-331.

Gibbs, K.A. et al., 2008. Genetic determinants of self identity and social recognition in bacteria. Science 321, 256-259.

Glass, N.L., Dementhon, K., 2006. Non-self recognition and programmed cell death in filamentous fungi. Curr. Opin. Microbiol. 9, 553-558.

Glass, N.L. et al., 2000. The genetics of hyphal fusion and vegetative incompatibility in filamentous ascomycete fungi. Annu. Rev. Genet. 34, 165-186.

Grafen, A., 1990. Do animals really recognize kin? Anim. Behav. 39, 42-54.

Grosberg, R.K., 1987. Limited dispersal and proximity-dependant mating success in the colonial ascidian Botryllus schlosseri. Evolution 41, 372-384.

Grosberg, R.K., 1988. The evolution of allorecognition specificity in clonal invertebrates. Quart. Rev. Biol. 63, 377-412.

Grosberg, R.K., Quinn, J.F., 1986. The genetic control of kin recognition by the larvae of a colonial marine invertebrate. Nature 322, 456-459.

Grosberg, R.K., Quinn, J.F., 1989. The evolution of selective aggression conditioned on allorecognition specificity. Evolution 43, 504-515.

Grosberg, R.K., Strathmann, R.R., 2007. The evolution of multicellularity: a minor major transition? Ann. Rev. Ecol., Evol., Syst. 38, 621-654.

Hamilton, W.D., 1964. Genetical evolution of social behaviour I+II. J. Theor. Biol. 7, $1-34$

Hamilton, W.D., May, R.M., 1977. Dispersal in stable habitats. Nature 269, 578-581.

Hartl, D.L. et al., 1975. Adaptive significance of Vegetative Incompatibility in Neurospora crassa. Genetics 81, 553-569.

Hirose, S. et al., 2011. Self-recognition in social amoebae is mediated by allelic pairs of tiger genes. Science 333, 467-470.

Holman, L. et al., 2013. Crozier's paradox revisited: maintenance of genetic recognition systems by disassortative mating. BMC Evol. Biol. 13, pp. 211-211.

Hughes, R.N. et al., 2004. Kin or self-recognition? Colonial fusibility of the bryozoan Celleporella hyalina. Evol. Dev. 6, 431-437.

Ispolatov, I. et al., 2012. Division of labour and the evolution of multicellularity. Proc. Roy. Soc. B-Biol. Sci. 279, 1768-1776.

Jansen, V.A.A., van Baalen, M., 2006. Altruism through beard chromodynamics. Nature 440, 663-666.

Jost, L., 2006. Entropy and diversity. Oikos 113, 363-375.

Koschwanez, J.H. et al., 2011. Sucrose utilization in budding yeast as a model for the origin of undifferentiated multicellularity. PLOS Biol. 9.
Kuzdzal-Fick, J.J. et al., 2011. High relatedness is necessary and sufficient to maintain multicellularity in Dictyostelium. Science 334, 1548-1551.

Lacey, J., 1996. Spore dispersal - its role in ecology and disease: the British contribution to fungal aerobiology. Mycol. Res. 100, 641-660.

Laird, D.J. et al., 2005. Stem cells are units of natural selection in a colonial ascidian. Cell 123, 1351-1360.

Lee, W. et al., 2012. An evolutionary mechanism for diversity in siderophoreproducing bacteria. Ecol. Lett. 15, 119-125.

Nauta, M.J., Hoekstra, R.F., 1994. Evolution of vegetative incompatibility in filamentous ascomycetes. 1. Deterministic models. Evolution 48, 979-995.

Okasha, S., 2006. Evolution and the Levels of Selection. Oxford University Press, Oxford.

Ostrowski, E.A. et al., 2008. Kin discrimination increases with genetic distance in a social amoeba. PLOS Biol. 6, 2376-2382.

Paoletti, M., Saupe, S.J., 2009. Fungal incompatibility: evolutionary origin in pathogen defense? Bioessays 31, 1201-1210.

Pittenger, T., Brawner, T.G., 1961. Genetic control of nuclear selection in Neurospora heterokaryons. Genetics 46, 1645-\&.

Pontecorvo, G., 1958. Trends in Genetic Analysis New York. Columbia University Press, p. 145.

Queller, D.C., Strassmann, J.E., 2009. Beyond society: the evolution of organismality Philos. Trans. Roy. Soc. B-Biol. Sci. 364, 3143-3155.

Raymundo, L.J., Maypa, A.P., 2004. Getting bigger faster: mediation of size-specific mortality via fusion in juvenile coral transplants. Ecol. Appl. 14, 281-295.

Rayner, A.D.M., 1991. The challenge of the individualistic mycelium. Mycologia 83, 48-71.

Rayner, A.D.M. et al., 1984. The biological consequences of the individualistic mycelium. In: Jennings, D.H., Rayner, A.D.H. (Eds.), The Ecology and Physiology of the Fungal Mycelium. Cambridge University Press, Cambridge, pp. 509-540

Read, N., et al., 2010. Hyphal Fusion. In: Borkovich, K.A.D.E., (Ed.), Cellular and Molecular Biology of Filamentous Fungi American Society of Microbiology. pp. 260-273.

Richard, F. et al., 2012. Cooperation among germinating spores facilitates the growth of the fungus, Neurospora crassa. Biol. Lett. 8, 419-422.

Roca, M.G. et al., 2005. Conidial anastomosis tubes in filamentous fungi. Fems Microbiol. Lett. 249, 191-198.

Roper, M. et al., 2011. Nuclear and genome dynamics in multinucleate ascomycete fungi. Curr. Biol. 21, R786-R793.

Rousset, F., Roze, D., 2007. Constraints on the origin and maintenance of genetic kin recognition. Evolution 61, 2320-2330.

Saupe, S.J., 2000. Molecular genetics of heterokaryon incompatibility in filamentous ascomycetes. Microbiol. Mol. Biol. Rev. 64, 489-502.

Saupe, S.J. et al., 2000. Vegetative incompatibility in filamentous fungi: Podospora and Neurospora provide some clues. Curr. Opin. Microbiol. 3, 608-612.

Stoner, D.S., Weissman, I.L., 1996. Somatic and germ cell parasitism in a colonial ascidian: possible role for a highly polymorphic allorecognition system. Proc Natl. Acad. Sci. USA 93, 15254-15259.

Stoner, D.S. et al., 1999. Heritable germ and somatic cell lineage competitions in chimeric colonial protochordates. Proc. Natl. Acad. Sci. USA 96, 9148-9153.

Strassmann, J.E. et al., 2011. Kin discrimination and cooperation in microbes. Ann. Rev. Microbiol. 65 (65), 349-367.

Tsutsui, N.D., 2004. Scents of self: the expression component of self/nonself recognition systems. Ann. Zool. Fenn. 41, 713-727.

van Zweden, J., d'Ettorre, P., 2010. Nestmate recognition in social insects and the role of hydrocarbons. In: AG, B., GJ, B. (Eds.), Insect Hydrocarbons: Biology, Biochemistry and Chemical Ecology. Cambridge University Press, Cambridge, pp. 222-243.

Vos, M., Velicer, G.J., 2008. Isolation by distance in the spore-forming soil bacterium Myxococcus xanthus. Curr. Biol. 18, 386-391.

West, S.A. et al., 2007. Evolutionary explanations for cooperation. Curr. Biol. 17 R661-R672.

Wilson, A.C.C., Grosberg, R.K., 2004. Ontogenetic shifts in fusion-rejection thresholds in a colonial marine hydrozoan, Hydractinia symbiolongicarpus. Behav. Ecol. Sociobiol. 57, 40-49.

Wolfenbarger, D.O., 1946. Dispersion of small organisms - distance dispersion rates of bacteria, spores, seeds, pollen and insects - incidence rates of disease and injuries. Am. Midland Nat. 35, 1-152.

Xiang, Q.J. et al., 2002. The ham-2 locus, encoding a putative transmembrane protein, is required for hyphal fusion in Neurospora crassa. Genetics 160, 169180. 